\title{
Metal-Organic Frameworks as Potential Drug Carriers
}

\author{
Rachel C. Huxford, Joseph Della Rocca, and Wenbin Lin \\ Department of Chemistry, CB\#3290, University of North Carolina, Chapel Hill, NC 27599 (USA) Fax: \\ 919-962-2388 Tel: 919-962-6320 \\ Wenbin Lin: wlin@unc.edu
}

\section{Summary of Recent Advances}

\begin{abstract}
Nanoparticle-based therapeutics have received increasing attention, as these systems can alleviate many drawbacks of conventional therapy. Metal-organic frameworks (MOFs), a new class of hybrid materials composed of metal ions and organic bridging ligands, have emerged as a promising platform for drug delivery, owing to their high drug loadings, biodegradability, and versatile functionality. The bulk MOF materials can absorb and release large amounts of therapeutics including ibuprofen, procainamide, and nitric oxide. Scale-down of MOFs to the nano-regime yields nanoscale metal-organic frameworks (NMOFs) which are more applicable as delivery vehicles, such as selective delivery of cisplatin prodrugs. Although progress has been made in utilizing NMOFs for drug delivery, many improvements must occur before they can become viable nanotherapeutics.
\end{abstract}

\section{Introduction}

Despite remarkable progress in our understanding of the fundamental biology behind many diseases, we have yet to observe comparable advances in the treatment of these diseases. Current therapeutics are limited by their nonspecific distribution throughout the body leading to high doses, rapid clearance, poor pharmacokinetics, and high side effects [1,2]. While a number of different strategies have been developed to improve the efficacy of conventional drugs, nanoparticle-based therapeutics have received an increasing amount of attention over the past 20 years. Many of the drawbacks of small molecule drugs can be alleviated through use of these novel systems. Typically, a nanoparticle therapeutic is composed of an active agent incorporated within the nanoparticle carrier; a variety of nanocarriers have been used for this purpose, such as polymeric nanoparticles, micelles, liposomes, iron oxide, and gold [3,4]. Some of the key properties of nanomaterials are small size, high drug loading, surface properties, drug release kinetics, improved pharmacokinetics, and biocompatibility [5-8]. Additionally, nanoparticles can be specifically targeted to diseased tissues (e.g., tumor regions) by conjugation with targeting ligands and can be engineered to contain multiple agents (e.g., imaging and therapeutic agents for both imaging and therapy), both of which are much harder to achieve with conventional therapeutics. The clinical success of nanoparticle therapies such as Abraxane and Doxil illustrates the power of this approach. A large number of promising nanoparticle-drug conjugates are currently in different stages of clinical development $[1,3,4]$.

Metal-organic frameworks (MOFs) are a new class of highly tunable hybrid materials crafted from metal connecting points and organic bridging ligands [9]. They are typically synthesized

Correspondence to: Wenbin Lin, wlin@unc. edu.

Publisher's Disclaimer: This is a PDF file of an unedited manuscript that has been accepted for publication. As a service to our customers we are providing this early version of the manuscript. The manuscript will undergo copyediting, typesetting, and review of the resulting proof before it is published in its final citable form. Please note that during the production process errors may be discovered which could affect the content, and all legal disclaimers that apply to the journal pertain. 
under mild conditions via coordination-directed self-assembly processes (Figure 1), and are also called coordination polymers or coordination networks[10-14]. Known for their large porosity with tunable pore sizes, shapes, and functionalities, MOFs are well studied among the scientific community for many applications such as nonlinear optics, gas storage, catalysis, and chemical sensing [9,10,13-20]. MOFs exhibit many desired characteristics as drug carriers, including exceptionally high surface areas and large pore sizes for drug encapsulation, intrinsic biodegradability as a result of relatively labile metal-ligand bonds, and versatile functionality for postsynthetic grafting of drug molecules. Over the past three years, MOFs have been investigated for applications in loading and release of several drug molecules. MOFs can be scaled down to the nano-regime to form nanoscale metal-organic frameworks (NMOFs) by using a variety of different techniques that have been developed for inorganic and organic polymeric nanoparticles. NMOFs are potential nanovectors for delivering therapeutic agents to targeted areas of the body, as they are able to control drug release with their large surface areas, high porosity, and presence of functional groups to interact with loaded moieties. Our group has recently reported MOFs as important delivery devices for contrast enhancement agents for magnetic resonance imaging, computed tomography, and optical imaging [21-25]; however, this review will focus on applications of MOFs and NMOFs as potential drug carriers. Although still at its infancy, NMOFs exhibit many desired characteristics as nanocarriers; intrinsic tunability and biodegradability as well as exceptionally high drug loading capacity of this emerging class of hybrid nanomaterials should make them a promising candidate for further development as nanotherapeutics.

\section{Proof of Concept: Drug Release with MOFs}

In developing MOFs for drug delivery, the goal is to design carriers that show little toxicity in the body; biocompatibility of both the metal and bridging ligand must be considered. While chromium and other metals are highly toxic, some metals exist in appreciable amounts in the body. For example, iron is a component in hemoglobin and is approximately $22 \mu \mathrm{M}$ in blood plasma. Tissue also contains various metals, such as copper $(68 \mu \mathrm{M})$, manganese $(180 \mu \mathrm{M})$, nickel $(2 \mu \mathrm{M})$, and zinc $(180 \mu \mathrm{M})$ [26]. Since this is an emerging class of drug carriers, there is very limited data on the biological fate of these systems. Iron oxide nanoparticles are clinically approved as MRI contrast agents, and in vitro assays have shown that these particles do not exhibit toxicity [27].

The first group of MOFs to be investigated as a potential drug delivery system is the MIL (Materials of Institut Lavoisier) family, pioneered by Férey and co-workers [28]. This investigation occurred at about the same time as the Lin group first explored the applications of NMOFs as potential MRI contrast agents [22]. The MIL family of MOFs is crafted from trivalent metal centers and carboxylic acid bridging ligands. The MIL family holds great promise in drug delivery for their attractive characteristics: large pores (25-34 $\AA$ ), outstanding surface areas $\left(3100-5900 \mathrm{~m}^{2} / \mathrm{g}\right)$, and the ability to incorporate functional groups into the framework.

Férey and co-workers studied the storage and release of Ibuprofen with chromium-based MIL-101 (Figure 2) and MIL-100 [29]. Both materials showed high Ibuprofen loading, with $0.347 \mathrm{~g}$ Ibuprofen $/ \mathrm{g}$ MOF for MIL-100 and $1.376 \mathrm{~g}$ Ibuprofen/g MOF for MIL-101. The drastic difference in drug loading between the two materials is attributed to the pore sizes of the materials; MIL-101 has larger pore volumes of 12700 and $20600 \AA$ (8200 and $12700 \AA$ for MIL-100). The kinetics of Ibuprofen release was investigated by suspending the Ibuprofenloaded materials in simulated body fluid (SBF) at $37{ }^{\circ} \mathrm{C}$. There is an initial release of weaklybound drug molecules within the first $2 \mathrm{~h}$ for MIL-100, and the entire cargo is released within 3 days. For MIL-101, steady release is observed for the first $8 \mathrm{~h}$ with complete release after 6 days. These MOFs contain toxic chromium, and thus, the use of these materials for drug 
delivery is very limited. A less-toxic analog, MIL-101(Fe) has been developed as a biocompatible alternative [30], and should be much more appropriate drug carriers.

The Férey group has also reported controlled drug release for MIL-53, a more flexible MOF in the MIL family [31]. Both MIL-53(Cr) and the less toxic MIL-53(Fe) achieved loadings of $0.220 \mathrm{~g}$ Ibuprofen/g MOF and $0.210 \mathrm{~g}$ Ibuprofen/g MOF, respectively. The delivery kinetics was investigated in SBF at $37^{\circ} \mathrm{C}$. Complete drug delivery occurred in 3 weeks, a long release time attributed to the flexibility of the MIL-53 framework and the strong drug-framework interactions. The prolonged drug release gives MIL-53 the potential of being used for sustained release and drug delivery. The drug loadings achievable with the MIL family are much higher than any material previously studied for drug encapsulation (i.e., through non-covalent interactions). Given the ability to construct highly porous MOFs with tunable hydrophobicity from practically any metal centers, we foresee many more porous MOFs will be examined as potential drug carriers.

The MOFs with hydrophobic pores such as the MIL family are ideal for encapsulating drug molecules that have poor aqueous solubility. MOFs can also be designed to have hydrophilic pores that can carry either positive or negative charges, and such MOFs can be used to encapsulate drugs that contain opposite charges to the MOFs. Rosi and co-workers recently developed an anionic MOF composed of zinc(II) ions, adenine, and para-biphenyldicarboxylic acid that can be cationically-triggered to release its cargo.[32] The MOF is anionic, so its use for storage and release of cationic drugs via exchange with cations in biological fluid was investigated. The MOF was loaded with $0.22 \mathrm{~g} / \mathrm{g}$ of hydrochloride salt of procainamide, an antiarrythmia drug. Procainamide therapy is currently limited by its rapid clearance from the body, requiring dosing every 3-4 hrs. The drug release behavior of the MOF was monitored by HPLC; complete release was observed at $72 \mathrm{~h}$ in phosphate buffered saline (PBS). In contrast, the drug-loaded MOF was also dialyzed against pure water, with only $20 \%$ of the drug released, suggesting that the majority of procainamide is released by the cations present in PBS. In addition, the authors demonstrated that the framework remained intact under these conditions.

MOFs have also been studied as carriers for gaseous therapeutics which represent a significant challenge for drug delivery. Nitric oxide (NO) has several applications in therapy, including antibacterial, antithrombotic, and wound-healing applications.[33,34] The storage and release of nitric oxide (NO) in a MOF was investigated by Morris and co-workers [35]. Two MOFs were synthesized by a previously reported procedure [36], from either cobalt or nickel and 2,5dihydroxyterephthalic acid. These MOFs can absorb 7 times the amount of NO than any previously reported material on a per gram basis via ligation to coordinatively unsaturated metal centers, with little background release [37]. Figure 3 depicts the activation, loading, and release of NO by these MOFs. The effect of the NO-loaded MOFs on precontracted pig coronary arteries was investigated. The MOFs resulted in more vessel relaxation than the NOfree control. While Ni and $\mathrm{Co}$ are too toxic to be used for biological applications, the work discussed thus far shows the ability of MOFs to absorb and release large amounts of therapeutic cargo. The ability to deliver significant doses of NO is also relevant to cancer therapy since NO has shown anticancer activity in high concentrations, but shows tumorigenic properties at lower concentrations. The concentration at which this switch occurs is unknown [38,39].

\section{Drug Delivery with Nano-MOFs}

While the systems discussed above illustrate the promise of MOFs as drug delivery vehicles, in vivo applications of the bulk MOFs are however limited because they are not suitable for systemic circulation. The bulk MOFs thus need to be scaled down to the nano-regime so that the resulting NMOFs can circulate systemically while maintain the advantageous properties 
of bulk MOFs for drug delivery. NMOFs can improve the pharmacokinetic properties of the encapsulated drugs $[1,5,7]$.

The Lin group was able to fabricate an NMOF (designated as NCP-1) from $\mathrm{Tb}^{3+}$ ions and $c, c, t$-(diamminedichlorodisuccinato) $\mathrm{Pt}(\mathrm{IV})$ (disuccinatocisplatin, DSCP), a cisplatin prodrug [40]. The NCP-1 nanoparticles were $58.3 \pm 11.3 \mathrm{~nm}$ in diameter, and were encapsulated with silica to enhance stability (half-life of $9 \mathrm{~h}$ in HEPES buffer at $37^{\circ} \mathrm{C}$, compared to $1 \mathrm{~h}$ for uncoated). Silica-coated NCP-1 particles were further functionalized with c(RGDfk), a cyclic peptide that targets the $\alpha_{v} \beta_{3}$ integrin, which is overexpressed in many cancers. The cytotoxicity of the nanoparticles was investigated against HT-29 human colon adenocarcinoma cells. These particles displayed a lower $\mathrm{IC}_{50}$ (Inhibitory Concentration, 50\%) than that of cisplatin (9.7 $\mu \mathrm{M}$ vs. $13.0 \mu \mathrm{M}$ for cisplatin), while the untargeted particle did not exhibit significant cell death. The improved cytotoxicity of the c(RGDfk)-functionalized particles suggests that these particles are taken up by receptor-mediated endocytosis, followed by reduction to the active $\mathrm{Pt}(\mathrm{II})$ species by the reducing environment inside the cell (Figure 4). The ability to switch from a relatively nontoxic prodrug to a highly potent anticancer drug in such a delivery scheme is very attractive since it facilitates selective delivery of anticancer drugs to cancerous cells, which can in turn lower the dose-limiting side effects that plague most anticancer chemotherapeutics.

Recently, the Lin group reported loading and release of biomedical agents in nanoMIL-101 (Fe).[41] This is the first report of the synthesis of an NMOF and subsequent loading of a fluorophore and anticancer agent via post-synthetic modification (Figure 5). The NMOF particles of MIL-101(Fe) were octahedral particles about $200 \mathrm{~nm}$ in diameter with impressive porosity, a Langmuir surface area of $3700-4535 \mathrm{~m}^{2} / \mathrm{g}$. Amino-functionalized nanoMIL-101 was synthesized by incorporating 2-aminoterephthalic acid in the synthesis. A Bodipy fluorophore was grafted on the NMOF through a covalent amine bond with up to $11 \mathrm{wt} \%$ loading. Confocal microscopy of the Bodipy-loaded particles with HT-29 cells showed fluorescent signal only with those cells incubated with the particles, indicating that the particles are able to cross the cell membrane and release their fluorescent cargoes. Additionally, a platinum(IV) prodrug, $c, c, t$ - $\left[\mathrm{PtCl}_{2}\left(\mathrm{NH}_{3}\right)_{2}(\mathrm{OEt})\left(\mathrm{O}_{2} \mathrm{CCH}_{2} \mathrm{CH}_{2} \mathrm{CO}_{2} \mathrm{H}\right]\right.$ (ethoxysuccinatocisplatin, ESCP) was grafted on amino nanoMIL-101, with an overall drug payload of $12.8 \mathrm{wt}$ $\%$. The particles were coated with silica to enhance stability in vitro, giving a half-life of $14 \mathrm{~h}$ in PBS at $37^{\circ} \mathrm{C}$, compared to $1.2 \mathrm{~h}$ for the uncoated particles. Silica-coated particles were grafted with c(RGDfk) and studies with the functionalized particles against HT-29 cells showed that the particles had comparable cytotoxicity to that of cisplatin $\left(\mathrm{IC}_{50}=21 \mu \mathrm{M}\right.$ for the particles, $\mathrm{IC}_{50}=20 \mu \mathrm{M}$ for cisplatin, $\mathrm{IC}_{50}=29 \mu \mathrm{M}$ for silica-coated particles only). Grafting of both an optical imaging agent and a cisplatin prodrug into the nanoMIL-101 system suggests the potential of using such a platform for theranostic applications that allow real-time monitoring of therapeutic responses of an anticancer drug.

It is worth noting that several related inorganic nanoparticle systems have also been developed as potential anticancer therapeutics. O'Halloran and co-workers were able to improve efficacy of the leukemia agent, $\mathrm{As}_{2} \mathrm{O}_{3}$ by formation of insoluble metal-arsenate complexes inside liposomes [42,43]. $\mathrm{As}_{2} \mathrm{O}_{3}$ is a potent therapy for leukemia, limited by its severe side effects and poor pharmacokinetics. Salts of nickel, cobalt, copper, and zinc were incorporated into liposomes, followed by loading of $\mathrm{As}(\mathrm{OH})_{3}$ to form insoluble $\mathrm{M}(\mathrm{II})$ complexes of $\mathrm{As}_{2} \mathrm{O}_{3}$. Folate-targeted liposomes with nickel/ $/ \mathrm{As}_{2} \mathrm{O}_{3}$ showed high cytotoxicity against $\mathrm{HeLa}$ cells with an $\mathrm{IC}_{50}$ of $1.8 \pm 0.6 \mu \mathrm{M}\left(\mathrm{IC}_{50}=4.3 \pm 0.3 \mu \mathrm{M}\right.$ for $\left.\mathrm{As}_{2} \mathrm{O}_{3}\right)$. Recently, O'Halloran and co-workers were able to coencapsulate $\mathrm{As}_{2} \mathrm{O}_{3}$ and a cisplatin-based drug (acetate salt of diaqua-cisplatin) inside a liposome using the same strategy [44]. This formulation exhibited comparable cytotoxicity to $\mathrm{As}_{2} \mathrm{O}_{3}$ and diaqua-cisplatin alone, with several different cell lines. Adair and coworkers were able to successfully encapsulate organic fluorophores and hydrophobic chemotherapeutics within calcium phosphate nanoparticles $[45,46]$. Calcium phosphate 
nanoparticles provide a $\mathrm{pH}$-tunable way to deliver encapsulated molecules to targeted cells with minimal background release. Ceramide, an apoptosis inducer, was encapsulated into the nanoparticles during synthesis. Preliminary in vitro cell assays against breast cancer and melanoma cell lines revealed that the nanoparticle conjugate showed highly effective induction of apoptosis, while the nanoparticle carrier displayed little toxicity. Additionally, the Ceramide-calcium phosphate conjugate showed activity in resistant cell lines. Although these systems are not NMOFs, they share some of the same design principles as NMOF-based nanotherapeutics.

\section{Conclusion}

Significant progress has been made in adapting MOFs and NMOFs for drug delivery. With these hybrid systems, an immense number of metal centers and organic building blocks can be pieced together and specifically tailored to form new materials with desirable characteristics as drug carriers. Since NMOFs represent an infinitely tunable material platform, many other drugs will be incorporated into them in a foreseeable future. The ability to carry both imaging and therapeutic agents in NMOFs should greatly facilitate the efficacy studies of this promising class of nanotherapeutics. The future for MOFs and NMOFs in drug delivery is bright, although many more improvements are needed before they can be considered for clinical applications.

\section{Acknowledgments}

We thank NIH (U54-CA119343) and NSF (DMR-0906662) for financial support and Dr. Liqing Ma for help with graphics.

\section{References}

1. Davis ME, Chen Z, Shin DM. Nanoparticle therapeutics: an emerging treatment modality for cancer. Nat Rev Drug Discovery 2008;7:771-782.

2. Dyson PJ, Sava G. Metal-based antitumour drugs in the post genomic era. Dalton Trans 2006:19291933. [PubMed: 16609762]

3. Cho K, Wang X, Nie S, Chen Z, Shin DM. Therapuetic nanoparticles for drug delivery in cancer. Clin Cancer Res 2008;15:1310-1316. [PubMed: 18316549]

4. Peer D, Karp JM, Hong S, Farokhzad OC, Margalit R, Langer R. Nanocarriers as an emerging platform for cancer therapy. Nat Nanotechnol 2007;2:751-760. [PubMed: 18654426]

5. Alexis F, Pridgen E, Molnar LK, Farokhzad OC. Factors affecting the clearance and biodistribution of polymeric nanoparticles. Mol Pharmaceutics 2008;5:505-515.

6. Ferrari M. Cancer nanotechnology: opportunities and challenges. Nat Rev Cancer 2005;5:161. [PubMed: 15738981]

7. Li SD, Huang L. Pharmcokinetics and biodistribution of nanoparticles. Mol Pharmaceutics 2008;5:496-504.

8. Sanhai WR, Sakamoto JH, Canady R, Ferrari M. Seven challenges for nanomedicine. Nat Nanotechnol 2008;3:242-244. [PubMed: 18654511]

9. Li H, Eddaoudi M, O'Keeffe M, Yaghi OM. Design and synthesis of an exceptionally stable and highly porous metal-organic framework. Nature 1999;402:276-279.

10. Evans OR, Xiong R-G, Wang Z, Wong GK, Lin W. Crystal engineering of acentric diamondiod metalorganic coordination networks. Angew Chem Int Ed 1999;38:536-538.

11. Moulton B, Zaworotko MJ. From molecules to crystal engineering: supramolecular isomerism and polymorphism in network solids. J Chem Rev 2001;101:1629-1658.

12. Kitagawa S, Kitaura R, Noro S-i. Functional porous coordination polymers. Angew Chem Int Ed 2004;43:2334-2375.

13. Oh M, Mirkin CA. Chemically tailorable colloidal particles from infinite coordination polymers. Nature 2005;438:651-654. [PubMed: 16319888] 
14. Evans OR, Lin W. Crystal engineering of NLO materials based on metal-organic coordination networks. Acc Chem Res 2002;35:511-522. [PubMed: 12118990]

15. Seo JS, Whang D, Lee H, Jun SI, Oh J, Jeon YJ, Kim K. A homochiral metal-organic porous material for enantioselective separation and catalysis. Nature 2000;404:982-986. [PubMed: 10801124]

16. Ferey G, Latroche M, Serre C, Millange F, Loiseau T, Percheron-Guegan A. Hydrogen adsorption in the nanoporous metal-benzenedicarboxylate $\mathrm{M}(\mathrm{OH})\left(\mathrm{O}_{2} \mathrm{C}-\mathrm{C}_{6} \mathrm{H}_{4}-\mathrm{CO}_{2}\right)\left(\mathrm{M}=\mathrm{Al}^{3+}, \mathrm{Cr}^{3+}\right), \mathrm{MIL}-53$. Chem Commun 2003:2976-2977.

17. Chen B, Wang L, Xiao Y, Fronczek FR, Xue M, Cui Y, Qian G. A luminescent metal-organic framework with Lewis basic pyridyl sites for the sensing of metal ions. Angew Chem Int Ed 2009;48:500-503.

18. Lan A, Li K, Wu H, Olsson DH, Emge TJ, Ki W, Hong M, Li J. A luminescent microporous metalorganic framework for the fast and reversible detection of high explosives. Angew Chem Int Ed 2009;48:2334-2338.

19. Lin W, Wang L, Ma L. A novel octupolar metal-organic NLO material based on a chiral 2D coordination network. J Am Chem Soc 1999;121:11249-11250.

20. Park KH, Jang K, Son SU, Sweigart DA. Self-supported organometallic rhodium quinonoid nanocatalysts for stereoselective polymerization of phenylacetylene. J Am Chem Soc 2006;128:8740-8741. [PubMed: 16819862]

21. Rieter WJ, Taylor KML, Lin W. Surface modification and functionalization of nanoscale metalorganic frameworks for controlled release and luminescence sensing. J Am Chem Soc 2007;129:9852-9853. [PubMed: 17645339]

22•. Rieter WJ, Taylor KML, An H, Lin W, Lin W. Nanoscale metal-organic frameworks as potential multimodal contrast enhancing agents. J Am Chem Soc 2006;128:9024-9025. To the best of our knowledge, this is the first report of the synthesis of an NMOF. NMOFs crafted from $\mathrm{Gd}^{3+}$ ions and benzenetricarboxylic acid (100 nm plates) displayed an improved water signal-enhancement than the clinically approved $\mathrm{T}_{1}$-weighted MR contrast agent OmniScan. [PubMed: 16834362]

23. Taylor KML, Rieter WJ, Lin W. Manganese-based nanoscale metal-organic frameworks for magnetic resonance imaging. J Am Chem Soc 2008;130:14358-14359. [PubMed: 18844356]

24. Taylor KML, Jin A, Lin W. Surfactant-assisted synthesis of nanoscale gadolinium metal-organic frameworks for potential multimodal imaging. Angew Chem Int Ed 2008;47:7722-7725.

25. deKrafft KE, Xie Z, Cao G, Tran S, Ma L, Zhou OZ, Lin W. Iodinated nanoscale coordination polymers as potential contrast agents for computed tomography. Angew Chem Int Ed. 2009 In Press.

26. Bertini, I.; Gray, HB.; Stiefel, EI.; Valentine, JS. Biological inorganic chemistry: structure and reactivity. Sausalito, CA: University Science books; 2007.

27. Park J, Yu MK, Jeong YY, Kim JW, Lee K, Phan VN, Jon S. Antibiofouling amphiphilic polymercoated superparamagnetic iron oxide nanoparticles: synthesis, characterization, and use in cancer imaging in vivo. J Mater Chem 2009;19:6412-6417.

28. Ferey G, Mellot-Draznieks C, Serre C, Millange F, Dutour J, Surble S, Margiolaki I. A chromium terephthalate-based solid with unusually large pore volumes and surface area. Science 2005;309:2040-2042. [PubMed: 16179475]

29•. Horcajada P, Serre C, Vallet-Regi M, Sebban M, Taulelle F, Ferey G. Metal-organic frameworks as efficient materials for drug delivery. Angew Chem Int Ed 2006;45:5974-5978. This work outlines the concept of using MOF materials for drug delivery for the first time. MIL-101(Cr) was successfully loaded with ibuprofen, and the release kinetics were investigated. This material displayed controlled release over 6 days.

30. Bauer S, Serre C, Devic T, Horcajada P, Morrot J, Ferey G, Stock N. High-throughput assisted rationalization of the formation of metal organic frameworks in the iron(III) aminoterephthalate solvothermal system. Inorg Chem 2008;47:7568-7576. [PubMed: 18681423]

31. Horcajada P, Serre C, Maurin G, Ramsahye NA, Balas F, Vallet-Regi M, Sebban M, Taulelle F, Ferey G. Flexible porous metal-organic frameworks for a controlled drug delivery. J Am Chem Soc 2008;130:6774-6780. [PubMed: 18454528]

32. An J, Geib SJ, Rosi NL. Cation-triggered drug release from a porous zinc-adeninate metal-organic framework. J Am Chem Soc 2009;131:8376-8377. [PubMed: 19489551] 
33. Miller MR, Megson IL. Recent developments in nitric oxide donor drugs. Br J Pharmacol 2007;151:305-321. [PubMed: 17401442]

34. Zhu HF, Ka B, Murad F. Nitric oxide accelerates the recovery from burn wounds. World J Surg 2007;31:624-631. [PubMed: 17308846]

35•. McKinlay AC, Ziao B, Wragg DS, Wheatley PS, Megson IL, Morris RE. Exceptional behavior over the whole adsorption-storage-delivery cycle for NO in porous metal organic frameworks. J Am Chem Soc 2008;130:10440-10444. This work displays the application of Co- and Ni-MOFs for application in nitric oxide (NO) storage. NO is a biologically important gas that has antibacterial, antithrombotic, and wound-healing properties. The authors demonstrated that these MOFs can store $\sim 7$ times the amount of NO than previously reported materials and release NO in a controlled fashion. [PubMed: 18627150]

36. Dietzel PDC, Morita Y, Blom R, Fjellvag H. In situ high-temperature single-crystal investigation of a dehydrated metal-organic framework compound and field-induced magnetization of onedimensional metal-oxygen chains. Angew Chem Int Ed 2005;44:6354-6358.

37. Wheatley PS, Butler AR, Crane MS, Fox S, Xiao B, Rossi AG, Megson IL, Morris RE. NO-releasing zeolites and their antithrombotic properties. J Am Chem Soc 2006;128:502-509. [PubMed: 16402837]

38. Sonveaux P, Jordan BF, Gallez B, Feron O. Nitric oxide delivery to cancer: why and how? Eur J Cancer 2009;45:1352-1369. [PubMed: 19153039]

39. Huerta S, Chilka S, Bonavida B. Nitric oxide donors: novel cancer therapeutics (review). Int J Oncol 2008;33:909-927. [PubMed: 18949354]

40• Reiter WJ, Pott KM, Taylor KML, Lin W. Nanoscale coordination polymers for platinum-based anticancer drug delivery. J Am Chem Soc 2008;130:11584-11585. NMOFs consisting of $\mathrm{Tb}^{3+}$ ions and a cisplatin prodrug were encapsulated in a silica shell and conjugated to the targeting peptide, $\mathrm{c}$ (RGDfk). These nanoparticles showed excellent cytotoxicity against the angiogenic colon adenocarcinoma cell line, HT-29, cytotoxicity comparable to that of cisplatin. [PubMed: 18686947]

41. Taylor-Pashow KML, Della Rocca J, Xie Z, Tran S, Lin W. Postsynthetic modifications of ironcarboxylate nanoscale metal-organic frameworks for imaging and drug delivery. J Am Chem Soc 2009;131:14261-14263. [PubMed: 19807179]

42. Chen H, Ahn R, Van den Bossche J, Thompson DH, O'Halloran TV. Folate-mediated intracellular drug delivery increases the anticancer efficacy of nanoparticulate formulation of arsenic trioxide. Mol Cancer Ther 2009;8:1955-1963. [PubMed: 19567824]

43. Chen H, MacDonald RC, Li S, Krett NL, Rosen ST, O'Halloran TV. Lipid encapsulation of arsenic trioxide attenuates cytotoxicity and allows for controlled anticancer drug release. J Am Chem Soc 2006;128:13348-13349. To increase the pharmacokinetics and biocompatibility of the leukemia agent arsenic trioxide $\left(\mathrm{As}_{2} \mathrm{O}_{3}\right)$, O'Halloran and coworkers designed liposomal formulations of the acetate salts of nickel, cobalt, copper, and zinc. When $\mathrm{As}_{2} \mathrm{O}_{3}$ is added to the formulations, it coordinates to the metals, forming a more biocompatible means of delivering this anticancer agent. The liposomes displayed appreciable cytotoxicity against a number of cancer cell lines. [PubMed: 17031934]

44. Chen H, Pazicni S, Krett NL, Ahn RW, Penner-Hahn JE, Rosen ST, O'Halloran TV. Coencapsulation of arsenic- and platinum-based drugs for targeted cancer treatment. Angew Chem Int Ed 2009;48:9295-9299.

45. Kester M, Heakal Y, Fox T, Sharma A, Robertson GP, Morgan TT, Altinoglu EI, Tabakovic A, Parette MR, Rouse SM, et al. Calcium Phosphate Nanocomposite Particles for In Vitro Imaging and Encapsulated Chemotherapeutic Drug Delivery to Cancer Cells. Nano Letters 2008;8:4116-4121. [PubMed: 19367878]

46. Morgan TT, Muddana HS, Altinoglu EI, Rouse SM, Tabakovic A, Tabouillot T, Russin TJ, Shanmugavelandy SS, Butler PJ, Eklund PC, et al. Encapsulation of Organic Molecules in Calcium Phosphate Nanocomposite Particles for Intracellular Imaging and Drug Delivery. Nano Letters 2008;8:4108-4115. [PubMed: 19367837] 

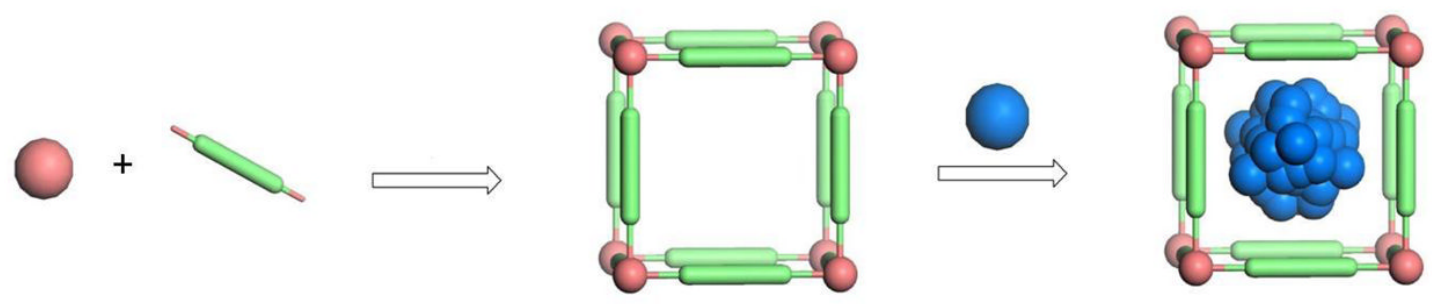

Figure 1.

Formation of MOFs by coordination-directed self-assembly processes and the loading of drugs into MOFs via physical encapsulation. Only one unit of an infinite MOF framework is shown for clarity. 


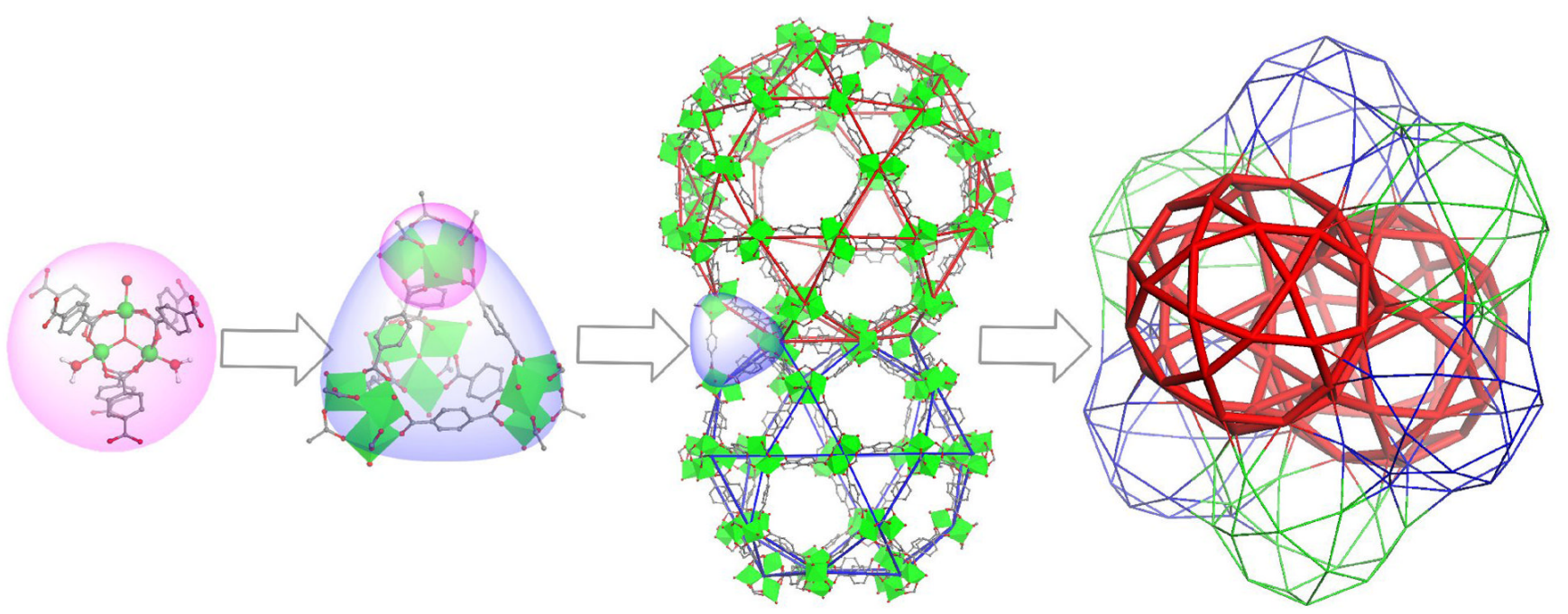

Figure 2.

The structure of MIL-101. The tetrahedra are assembled from trivalent metal centers $(\mathrm{Cr}$ or $\mathrm{Fe}$ ) and 1,4-benzenedicarboxylate. MIL-101 possesses larger cages than MIL-100, which facilitates Ibuprofen loading. 


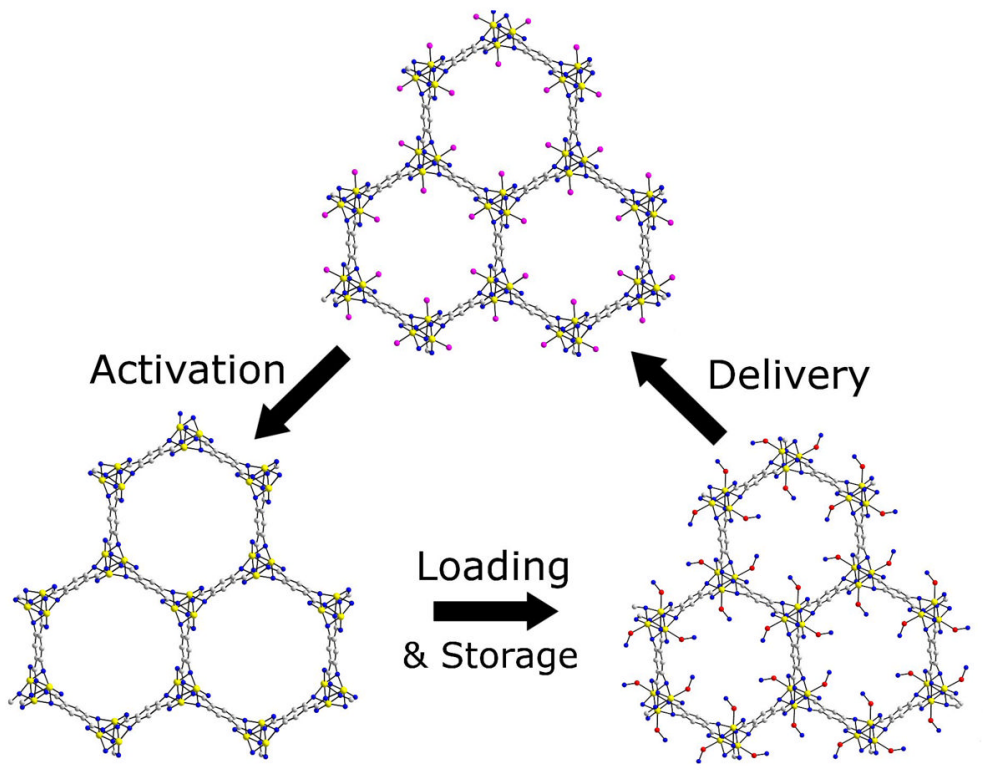

Figure 3.

Absorption, storage, and release of nitric oxide (NO) in a Co or Ni MOF. Activation occurs by dehydration of the MOF at $110^{\circ} \mathrm{C}$, followed by $\mathrm{NO}$ loading at room temperature. Finally, delivery (release) of $\mathrm{NO}$ is triggered when the MOF was exposed to $11 \%$ relative humidity. (Pink spheres indicate water molecules, red and blue spheres indicate nitric oxide.) 
A)
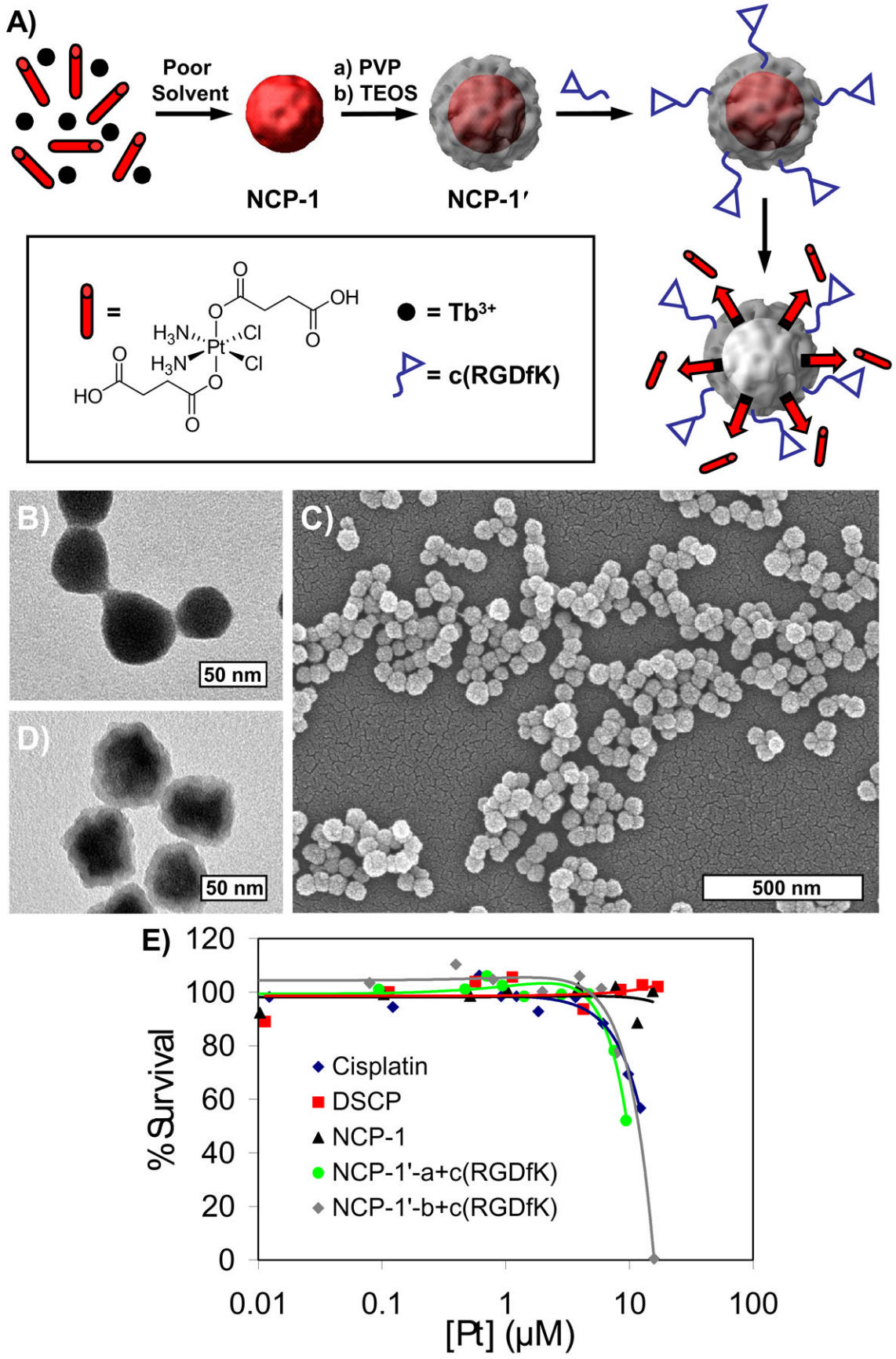

Figure 4.

A) Schematic showing the synthesis of Tb-DSCP NMOF (designated as NCP-1) and its subsequent coating with silica shell (NCP-1') and conjugation with cyclic peptide. (PVP = polyvinylpyrollidone, TEOS = tetraethylorthosilicate) B) TEM micrograph for as-synthesized NCP-1. C) TEM and D) SEM micrographs for NCP-1'. E) In vitro cytotoxicity assay curves for HT-29 cells obtained by plotting the \% cell viability against the Pt concentration of various samples and cisplatin control. 


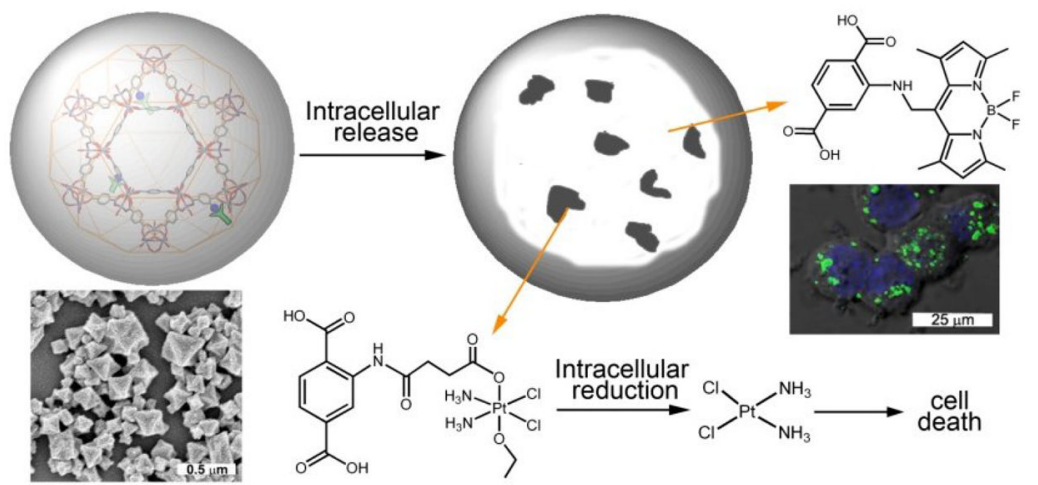

Figure 5.

NanoMIL-101 can be loaded both with an optical imaging agent and cisplatin prodrug. Simultaneous release of the fluorophore and cisplatin prodrug allows real-time monitoring of the drug delivery by optical imaging. 